\title{
Detection of the sign of expansion as a function of field size and eccentricity
}

\author{
SUSAN F. TE PAS, ASTRID M. L. KAPPERS, and JAN J. KOENDERINK \\ Universiteit Utrecht, Utrecht, The Netherlands
}

\begin{abstract}
Small optical flow fields are involved in object and shape recognition. These tasks depend on high resolution and low speed of the flow-field elements. Large optical flow fields are involved in tasks such as orientation and navigation, which require flow-field elements to move at high speed. From the above, we would expect to find different behavior of subjects for different parameter ranges of field size and speed of the elements. In this paper, we address the question of whether such different behavior exists for a task that is associated neither with object recognition nor with navigation. We obtained detection thresholds for expansion in the presence of translation for a wide range of field sizes. The same paradigm was used to investigate whether subjects made efficient use of peripheral information. We found viewing-distance invariance, meaning that subjects' performance scaled with stimulus size. Subjects performed very similarly with and without foveal information.
\end{abstract}

The optical flow field (Gibson, 1950, 1966) is a rich source of information about the structure of the world around us and our movement relative to it. An important question in psychophysics is how efficiently the visual system extracts this information, and whether this extraction depends on the task at hand (Koenderink \& van Doorn, 1975,1976 ).

Small fields of view (a few degrees of visual angle) are involved in object and shape recognition. Here, it seems likely that high resolution at low speeds is required, because objects will typically be tracked with pursuit eye movements. The shape and texture of the elements are probably also important. Performance should depend on first and higher order differential structure, since, for instance, surface curvature affects the second order flow structure (Koenderink, 1986; Koenderink \& van Doorn, 1975). Large fields of view (almost totally surrounding the subject) are important for detecting the general layout of a scene as well as for orientation and navigation in space. Here, resolution, shape, and texture of the elements are probably not important. Performance should depend mainly on the zeroth and first order structure of the flow field, at high speeds, since global flows typically cannot be cancelled by tracking eye movements (Koenderink, 1986; Koenderink \& van Doorn, 1975, 1976). Resolution is probably of minor importance for orientation and navigation. In view of the above, we would expect a priori qualitative differences in performance of subjects for different ranges of, for instance, field size, velocity, and eccentricity.

This research was supported by the Insight II Project of the ESPRIT basic research action of the European Commission and the Netherlands Organization for Scientific Research. We would like to thank H.-J. van Veen and $A$. van Pelt for their valuable contribution and S. McNab for correcting the English. Correspondence should be addressed to S. F. te Pas, Universiteit Utrecht, Helmholtz Instituut, Princetonplein 5, 3584 CC Utrecht, The Netherlands (e-mail: s.f.tepas@fys.ruu.nl).
Dijkstra, Cornilleau-Pérès, Gielen, and Droulez (1995) performed experiments with motion-defined curved surfaces (i.e., they used second order flow fields). They performed experiments with two different field sizes $\left(8^{\circ}\right.$ and $90^{\circ}$ of visual angle), and three different kinds of motion from which to derive the shape of the objects (object rotation, self-motion, and object translation). Although performance was similar for all three types of motion in the experiment with large fields of view, performance was best for object rotation, intermediate for self-motion, and worst for object translation in the experiments with small fields of view. Thus, we feel that such qualitative differences between small and large fields of view might also be expected in experiments with first-order flow fields.

On the other hand, several psychophysical experiments on zeroth-order flow fields suggest that motion detection in the periphery scales with the cortical magnification factor (McKee \& Nakayama, 1984; Orban, Van Calenbergh, De Bruyn, \& Maes, 1985; van de Grind, van Doorn, \& Koenderink, 1983). Physiological studies by Hubel and Wiesel (1974) indicate that the average receptive field size increases linearly with eccentricity. Moreover, span and delay of Reichardt-type correlators for motion are qualitatively similar, and over a large range are also quantitatively similar in the central and peripheral visual field (van Doorn \& Koenderink, 1982a, 1982b; Koenderink, van Doorn, \& van de Grind, 1985). Warren and Kurtz (1992) and Crowell and Banks (1993) showed that a heading task (depending on large fields of view) is just as accurate with a large field of view as it is with a relatively small field of view $\left(10^{\circ}\right)$. From these results, we might expect only quantitative differences in performance of subjects for different field sizes and eccentricity. However, that the differences are quantitative rather than qualitative might be caused by the fact that most investigations use zeroth-order flow fields in which there is no shape information present. The investigation" with first-order flow fields mostly ask for a 
heading direction judgment, which is of course a specific task for navigation.

In this paper, we specifically investigated field size and foveal versus peripheral information, because they are a priori different for object recognition versus navigation. We chose a first order differential structure because first order information is important for tasks requiring small as well as large fields of view. The same paradigm and stimulus were used for all field sizes. In this way, we ensure that all differences we find have been caused by the field size and not by the paradigm. In our first experiment, the polarity of divergence was detected in the presence of translational velocity at different field sizes (with a diameter of $3^{\circ}, 20^{\circ}$, and $140^{\circ}$ of visual angle). We varied the translational velocity that was present in the stimulus because we wanted to investigate whether the different speed ranges for large and small fields of view would cause qualitative differences in performance. We felt that the task of the subjects (determining the polarity of the divergence) would be associated with neither navigation nor object recognition.

In a second experiment, we tested whether subjects would make use of peripheral information. In this case we used a stimulus diameter of $140^{\circ}$ of visual angle only. To force subjects to use peripheral information, we introduced a central gap of variable size. In the past, several studies on peripheral vision have used a limited patch in the peripheral visual field (e.g., van Doorn \& Koenderink, 1982a, 1982b; McKee \& Nakayama, 1984; Orban et al., 1985; van de Grind et al., 1983), effectively moving the fixation point. In this way, one can conclude something about thresholds in peripheral versus foveal vision. However, in our experiments, peripheral information was always present. We changed the amount of foveal information to see if it is neccesary to perform the task. In the first experiment, we changed the field size, and with that, the amount of peripheral information. In this experiment, we kept the field size constant and varied the amount of foveal information.

\section{METHOD}

\section{Apparatus}

Our stimuli were generated on an Atari MegaST4 computer. For the smaller fields of view, they were shown on an Atari SM125 high-resolution white phosphor $\mathrm{P} 4$ monochrome monitor. The monitor dimensions were $13.6 \mathrm{~cm} \times 21.7 \mathrm{~cm}(400 \times 640$ pixels $)$. Subjects rested their heads on a chinrest, either 34 or $230 \mathrm{~cm}$ from the computer screen. Thus the screen area is either $22.6^{\circ} \times 35.4^{\circ}$ or $3.4^{\circ}$ $\times 5.4^{\circ}$ of visual angle. Pixel separation is $3.2^{\prime}$ or $0.3^{\prime}$ of arc. Each dot has a size of $3 \times 3$ pixels for the smaller fields of view and of 5 $\times 5$ pixels for the largest field of view to make sure that they were clearly visible in all locations of the screen. For the experiments with a large field of view, we used a translucent Barco projection screen that showed green light on a dark background (it was impossible to create white light). The screen dimensions were $250 \times 392 \mathrm{~cm}(400$ $\times 640$ pixels). Subjects again rested their heads on a chinrest $25 \mathrm{~cm}$ from the screen. The stimulus was projected from behind the translucent screen. Here, the screen area was almost a full hemisphere $\left(157.4^{\circ} \times 165.5^{\circ}\right)$ and subjects had the impression that they were totally surrounded by the stimulus. The refresh rate and update rate of both the Atari monitor and the Barco projection screen were $70 \mathrm{~Hz}$. The lifetime of the dots deteriorated to about 5 frames at the highest image velocities $\left(320^{\circ} / \mathrm{sec}\right.$ if the stimulus diameter was $\left.20^{\circ}\right)$, be- cause a dot doesn't stay inside the stimulus for the entire 16 frames. However, because we did not find effects of the lifetime of the dots in previous experiments (Kappers, van Doorn, \& Koenderink, 1994), we feel that this does not hamper performance. Also, subjects reported that they could see smooth motion at every velocity that we used in the experiments. Experiments were performed monocularly to avoid conflicting stereo cues, with all subjects using only their right eye. To avoid distractions from outside the stimulus, experiments were performed in a dark room. The only visible light came from either the computer screen or the projection screen.

\section{Stimulus}

Stimuli were similar to those used by Kappers et al. (1994) and te Pas, Kappers, and Koenderink (1996). We used pseudo-random dot patterns consisting of dark dots on a light background. The dots were situated on a dithered hexagonal point raster to eliminate obvious local features caused by (random) clustering. The stimulus window was circular with a diameter of 380 pixels $\left(3^{\circ}, 20^{\circ}\right.$, or $140^{\circ}$ of visual angle for the different field sizes). Moving patterns were generated by presenting sequences of frames stroboscopically. In between two consecutive frames, all dots "moved with the flow." The number of frames determined the total presentation time. We kept this constant at 16 frames $(228 \mathrm{msec}$ ). Previous experiments (Kappers et al., 1994) showed that the effect of the density and lifetime of the dots on performance was very small. Therefore we kept these variables constant at 64 dots per frame with a lifetime equal to the presentation time in this experiment. The number of dots was kept at 64 in the experiments with the central gap as well, although the dot density changed when a gap was added. However, we did some pilot experiments with different dot densities and performance remained the same over a wide range. Thus, we feel justified in comparing the results of the experiments with and without a central gap.

For stimulus movement, we chose a uniform expansion or contraction. In previous experiments, we found no differences at all between the detection of rotation, divergence, and deformation in the presence of translational velocity (te Pas et al., 1996). Because experiments are very time-consuming and we expected results to be similar for all first-order flow fields, we did not perform the experiments for rotation and deformation. By divergence, we mean the relative area expansion (or contraction) per second. A divergence of $2 / \mathrm{sec}$ means that an area of size $\mathrm{X}$ will have increased in $1 \mathrm{sec}$ by twice its own size to become $3 X$.

The center of the flow field (singular point or focus of expansion) was always located outside the stimulus window on a vertical axis through the center of the stimulus window, either below (for contraction) or above (for expansion) the fixation point. We achieved this by adding a translational velocity to the pure expansion field. In a pure expansion field, the center of the flow would be in the center of the stimulus. When we add a translational velocity, the center of the flow moves outward by an amount that is proportional to the amount of translational velocity. Figures $1 B-1 C$ show stimuli with two different values of the translational velocity, while the divergence is the same. Notice that when the translational velocity is higher, the center of the flow moves outward. We varied the speed of the translational velocity inside the stimulus window, although the direction of translational velocity was always downward. By adding a translational velocity, we change the size and the angular deviation of the vectors. We would like to stress that although this does mean a change in the zeroth-order optic flow field, the first-order flow field is not affected. In all conditions we have a constant divergence over the entire stimulus area. In this way, our stimuli differ from the ones used by Warren and Kurtz (1992). Because they used 3D clouds of dots, there was effectively a different value of the divergence at every point, whereas in our stimuli it was constant.

Figure IA shows an example of a single frame of a stimulus. Figure $1 \mathrm{~B}$ shows the 16 consecutive frames superimposed. Here the divergence is $1 / \mathrm{sec}$ and the translational velocity is $10 \% \mathrm{sec}$ for a field diameter of $20^{\circ}$ of visual angle. For a field diameter of $3^{\circ}$ or $140^{\circ}$, 

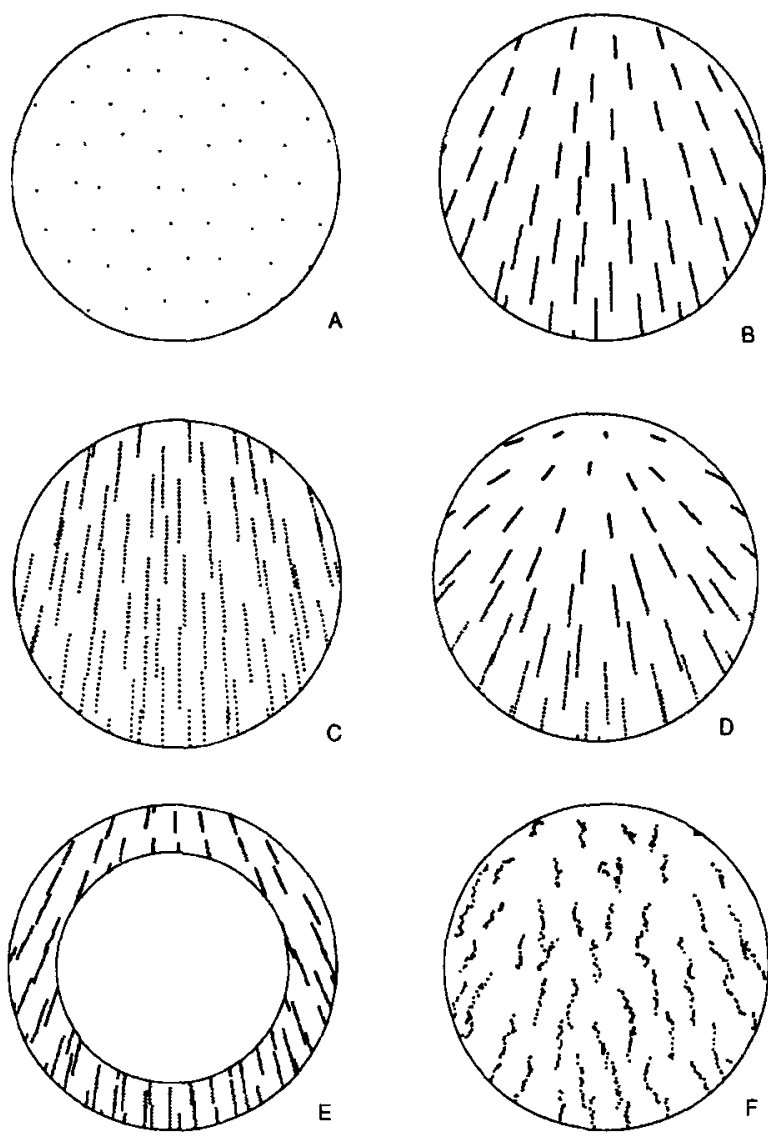

Figure 1. Different examples of the stimulus. For all examples, there are about 64 dots per frame and each dot has a lifetime of 16 frames. (A) A single frame of a stimulus. (B) 16 consecutive frames superimposed. The value of the divergence is $1 / \mathrm{sec}$ and the translational velocity is $10^{\circ} / \mathrm{sec}$ for a field size of $20^{\circ}\left(1.5^{\circ}\right.$ and $70^{\circ} / \mathrm{sec}$ for field sizes of $3^{\circ}$ and $140^{\circ}$, respectively). (C) The same as $B$, but with a translational velocity of $20 \% \mathrm{sec}$ for a field size of $20^{\circ}$. (D) The same as $B$, but with a divergence of $2 / \mathrm{sec}$. (E) The same as $B$, but with a central gap. (E) The same as $B$, but with a noise amplitude ( $S D$ of the Gaussian perturbation vector) of $0.1^{\circ}$ (for a field size of $20^{\circ}$ ).

this would mean a translational velocity of $1.5^{\circ}$ or $70^{\circ}$ per sec, respectively. Figures 1C-1D show stimuli with different values of the divergence and translational velocity.

In a second experiment, we used the large stimulus with a diam. eter of $140^{\circ}$ of visual angle, but this time we presented the stimuli with a central gap of variable size in order to force the subject to use peripheral information only. Figures $1 \mathrm{~B}$ and $1 \mathrm{E}$ show an example of the stimulus with and without the central gap. The value of the divergence and the translational velocity is the same in both figures and the 16 consecutive frames are superimposed. In the actual stimulus, we did not present borders like those depicted in Figure 1.

\section{Procedure}

In an experimental trial, we presented either an expansion or a contraction. Subjects had to indicate which of the two they had seen; that is, they had to determine the polarity of the divergence. Note that when we use the term divergence, or div, we actually mean the scalar quantity "divergence of the instantaneous velocity field." Psychophysical thresholds are measured by adding a stochastic noise component to the stimulus in the following manner: A two-dimensional
Gaussian perturbation vector with variable amplitude is added to each new position of the dots. As the amplitude increases, the dots will start to drift in a less deterministic manner (somewhat like Brownean diffusion). Note that the noise will affect both the direction and the magnitude of the velocity. Unfortunately, it is impossible to introduce noise that affects only the divergence, and not the translational velocity. In our experiments, both the div and the translational velocity were constant over the entire stimulus area. Therefore, we decided to use a uniform noise that would affect the position of the dots in the image.

At a certain noise level ( $S D$ of the Gaussian perturbation vector), the drift will be so large that the subject is unable to determine the polarity of the divergence. We use a two-alternative forced choice paradigm to determine noise thresholds using an adaptive staircase procedure. We start at zero noise level, and the first step size is $0.1^{\circ}$ for a stimulus diameter of $20^{\circ}$. We adaptively decrease the step size until a $75 \%$ correct threshold is reached. We have described this method extensively before (te Pas et al., 1996). Figure 1F shows a stimulus with a noise amplitude ( $S D$ of the Gaussian perturbation vector of $0.1^{\circ}$ for a stimulus diameter of $20^{\circ}$ ). All other conditions are the same as in Figure 1B. Before and after a trial, we presented a fixation cross in the middle of the stimulus area. Subjects were instructed to fixate during a trial.

Three subjects (one of the authors and two others) participated in the present experiments. Subject S.P. is emmetropic, Subjects H.V. and A.P. are myopic. Because glasses would distort stimuli with a diameter of $140^{\circ}$, both H.V. $(-2.5$ D) and A.P. $(-2$ D) were left uncorrected for these stimuli. For the smaller fields of view, we tested to see if there would be any change in performance for measurements with or without correction to normal. There turned out to be no difference whatsoever. Subject H.V. was corrected to normal for the experiments with diameters of $3^{\circ}$ and $20^{\circ}$ of visual angle. We left Subject A.P. uncorrected. Both H.V. and A.P. are experienced psychophysical observers who work in our lab. However, we did not inform them about the exact purpose of the experiments.

We sampled both the range of the translational velocity and the range of the flow component by doubling (or halving) their respective values until the subject was unable to perform the task even at zero noise level. For instance, for a divergence of $1 / \mathrm{sec}$ we obtained thresholds for a translational speed of $5^{\circ}, 10^{\circ}, 20^{\circ}, 40^{\circ}$, and so on, per sec. At a certain value, for instance, $80^{\circ} / \mathrm{sec}$, the subject could not perform the task at zero noise level. Then we would double the value of divergence and start all over again. Thus our data points span the entire range of combinations of divergence and translational speed the subject could measure, provided the center of divergence was kept outside the stimulus window. A typical experiment (see, for instance, Figure 2A) took about $4 \mathrm{~h}$.

\section{EXPERIMENT 1 Effects of Stimulus Size}

In our first experiment, we varied the stimulus size in order to investigate differences in performance for large and small optical flow fields. We used three different stimulus diameters, namely, $3^{\circ}, 20^{\circ}$, and $140^{\circ}$ of visual angle. Together they cover a vast range of stimulus sizes. Figures $2 \mathrm{~A}-2 \mathrm{C}$ show the noise level ( $S D$ of the Gaussian perturbation at $75 \%$ correct) as a function of translational velocity for Subject S.P. The field diameters are $3^{\circ}, 20^{\circ}$, and $140^{\circ}$ of visual angle, respectively. Results for other subjects were qualitatively the same. We were not primarily interested in the minor quantitative differences between subjects that occur in the height of the noise levels and the range that could be covered, so we will not show every result we obtained. We will present a representative selection 

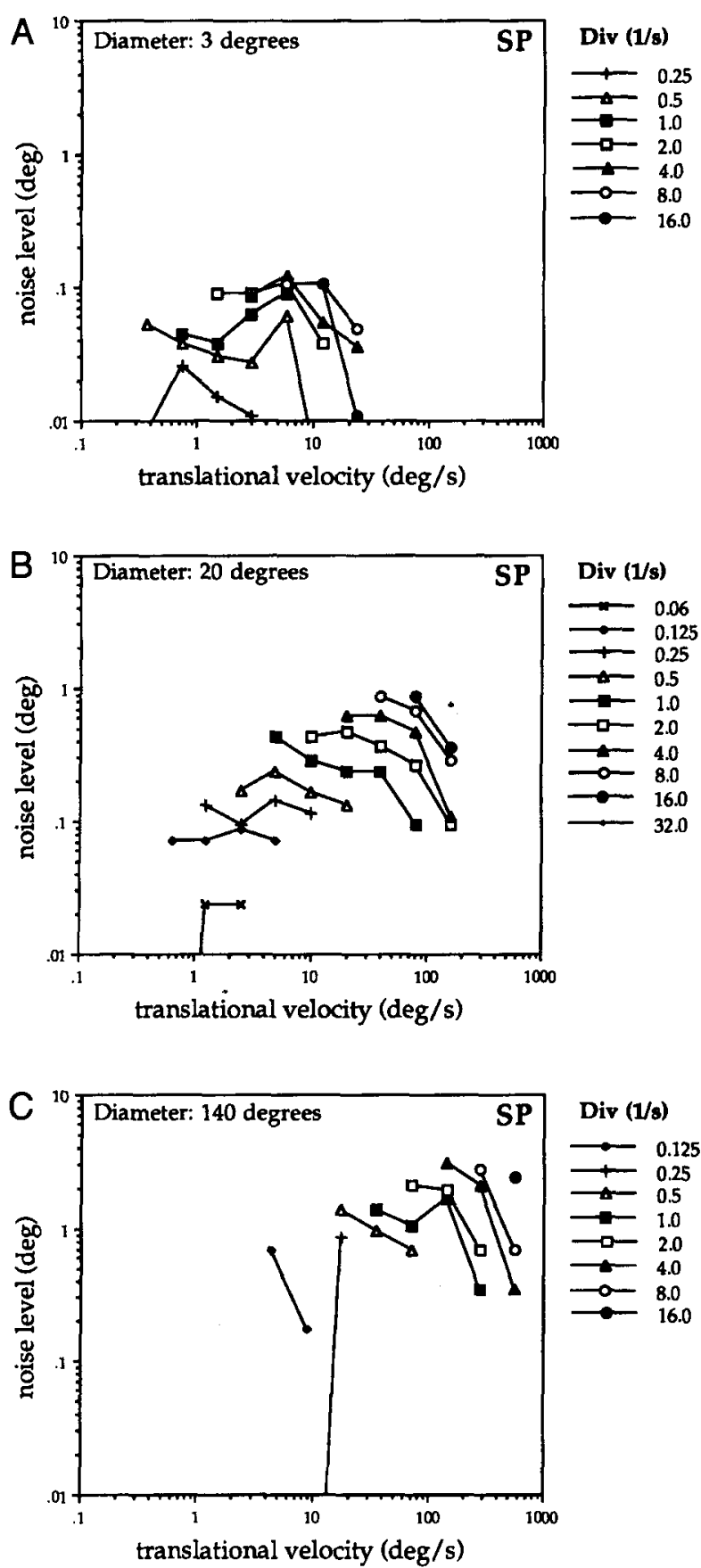

Figure 2. Noise levels ( $S D$ of the Gaussian perturbation vector) as a function of translational velocity for Subject S.P. for different field sizes. (A) A field size of $3^{\circ}$. (B) A field size of $20^{\circ}$. (C) A field size of $140^{\circ}$.

to illustrate our findings. One should bear in mind, however, that conclusions hold for the other subjects as well.

Although there were differences in the height of the noise levels that could be reached, qualitatively the graphs were very similar for all three diameters. We can characterize each curve by two parameters, a rather flat region where noise levels were independent of translational velocity, and a maximum translational velocity, above which subjects were unable to perform the task even at zero noise level. We have used this characterization before (Kappers et al., 1994; te Pas et al., 1996), and it turned out to be very useful. The characterization is shown schematically in Figure 3 . The point at the far left of each curve is forced by the experimental setup. It is the minimum translational velocity necessary to keep the center of divergence outside the stimulus window. To represent the rather flat region, we chose the maximum value of the curve. An alternative choice might have been the average height of the flat region, but it is difficult to define the exact end of the flat region. The point at the far right was determined by the performance of the subject. It represents the maximum value of the translational velocity at which the subject was still able to perform the task. Note that we actually measured the point beyond the end of the curve, but subjects couldn't even perform the task at zero noise level. All curves should therefore dive steeply to zero after the point at the far right. We omitted the steep dive because it tends to spoil the graph. The advantage of this method of characterization is that it condenses the data and yields a clear interpretation of the results.

Figures $4 \mathrm{~A}-4 \mathrm{~B}$ show the maximum noise level, as explained in Figure 3, as a function of divergence for Subjects S.P. and H.V., respectively. Thresholds depend on stimulus diameter and divergence in a characteristic manner. Notice that slopes are the same for all three stimulus sizes, suggesting that a change in stimulus diameter introduces only a shift in overall performance. To investigate the dependence of noise level on stimulus diameter, we plotted the same data in a different manner in Figures $5 \mathrm{~A}-5 \mathrm{~B}$. Here, we plotted the maximum noise level as a function of stimulus diameter. From these figures we can conclude that performance scales with stimulus diameter; in other words, we get the same results for all stimulus sizes if we divide thresholds by stimulus diameter. Again, all slopes are similar, about 0.8 for both S.P. and H.V. Subject A.P. measured curves for only two stimulus diameters

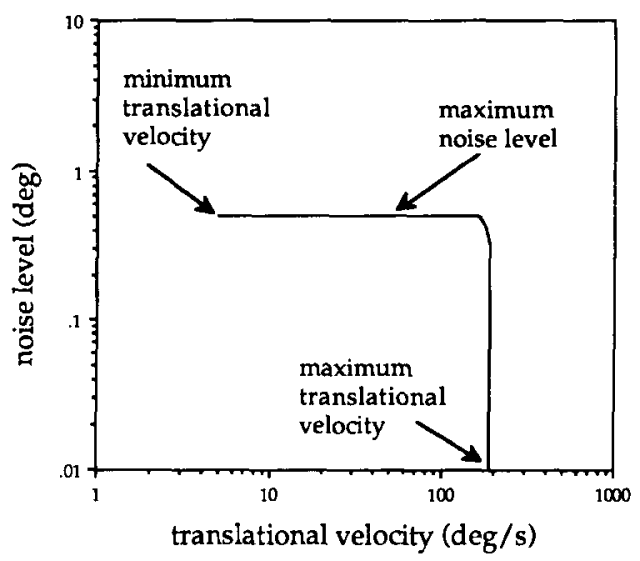

Figure 3. Representation of a typical curve for the noise level ( $S D$ of the Gaussian perturbation vector) at which subjects scored $\mathbf{7 5} \%$ correct as a function of the translational velocity. This curve can be characterized by a maximum noise level and a maximum translational velocity, as indicated. 

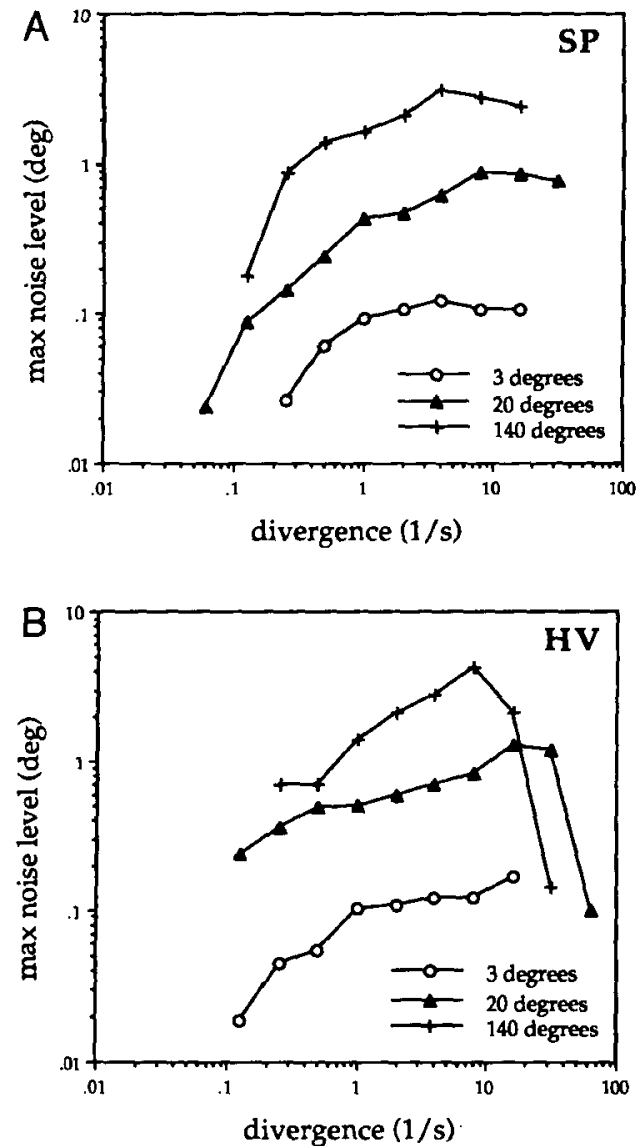

Figure 4. The maximum noise level (SD of the Gaussian perturbation vector) as a function of divergence for three different field sizes. (A) Results for Subject S.P. (B) Results for Subject H.V.

$\left(20^{\circ}\right.$ and $\left.140^{\circ}\right)$, but these two data points suggest a similar slope. Figures $6 \mathrm{~A}-6 \mathrm{~B}$ show the maximum translational velocity (see Figure 3) as a function of divergence for Subjects S.P. and H.V., respectively. Here we can also observe a shift in overall performance due to stimulus size. For S.P., the maximum translational velocity increased with divergence up to a certain point, then it saturated. For H.V., we also see an increase, although the saturation was much less clear.

\section{EXPERIMENT 2 Effects of Gap Size}

For our second experiment, we used only the large stimulus with a diameter of $140^{\circ}$ of visual angle. Here we used three different gap sizes of $0 \%, 25 \%$, and $50 \%$ of the stimulus area (gap diameters of $0^{\circ}, 107.8^{\circ}$, and $125.6^{\circ}$, respectively). The gap was situated in the middle of the stimulus. Of course, we could have increased the gap area even further, and at some point we would have found that performance deteriorated because there simply was not enough information in the stimulus. However, that was not what we were investigating. We wanted to find out what happens when there is enough information in the stimuli (al- though it varies somewhat due to different dot densities), but it is in different parts of the stimuli.

Results for subjects can again be characterized by a maximum noise level and a maximum translational velocity, as shown in Figure 3. Results for a large field with gap size $0 \%$ (no gap) are already plotted in Figure $2 \mathrm{C}$. Results for gaps of $25 \%$ and $50 \%$ of the stimulus area were very similar.

Because we used stimuli with central gaps for this experiment, we had the opportunity to situate the center of expansion inside the central gap. In this way, the point of zero speed remains invisible. For the largest gap size, we presented the center of expansion at half the radius, one quarter times the radius, and at the center of the stimulus. Therefore, we could measure a much broader range of translational velocities (from 0 up to $1,280 \% \mathrm{sec}$ ). The graphs that we obtained are characterized very well by the two parameters shown in Figure 3, indicating that the representation is a useful one for all field sizes.

Figures $7 \mathrm{~A}-7 \mathrm{~B}$ show the maximum noise level as a function of divergence for Subjects S.P. and H.V., respectively. The three different lines represent different gap sizes. From Figure 7, we can conclude that there is no effect of gap
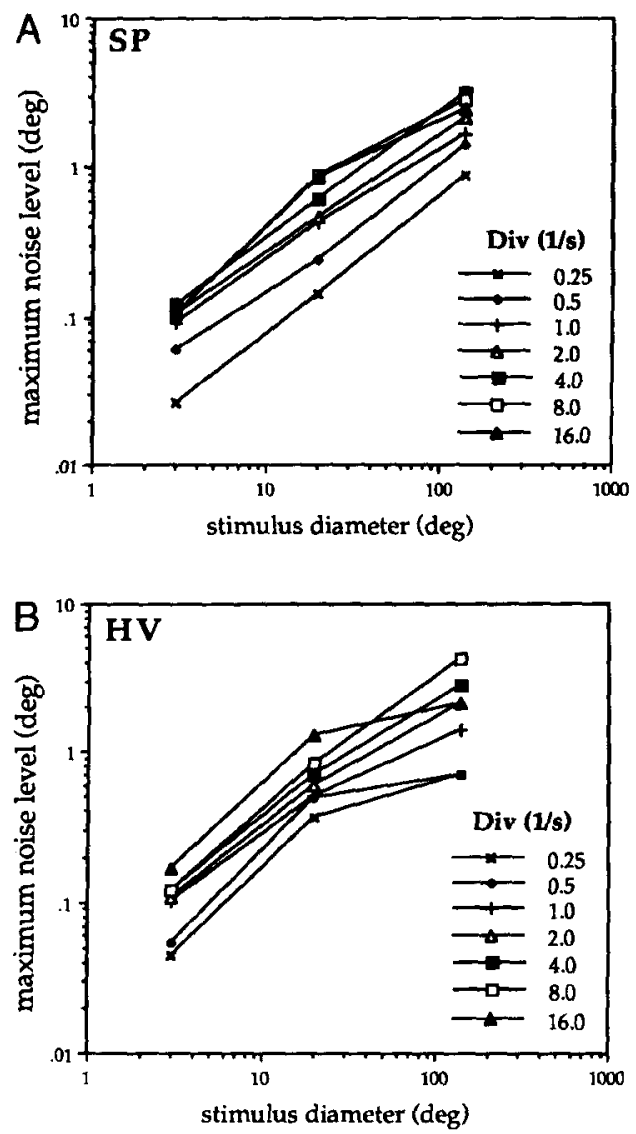

Figure 5. The maximum noise level ( $S D$ of the Gaussian perturbation vector) as a function of stimulus diameter for different values of the divergence. (A) Results for Subject S.P. (B) Results for Subject H.V. 

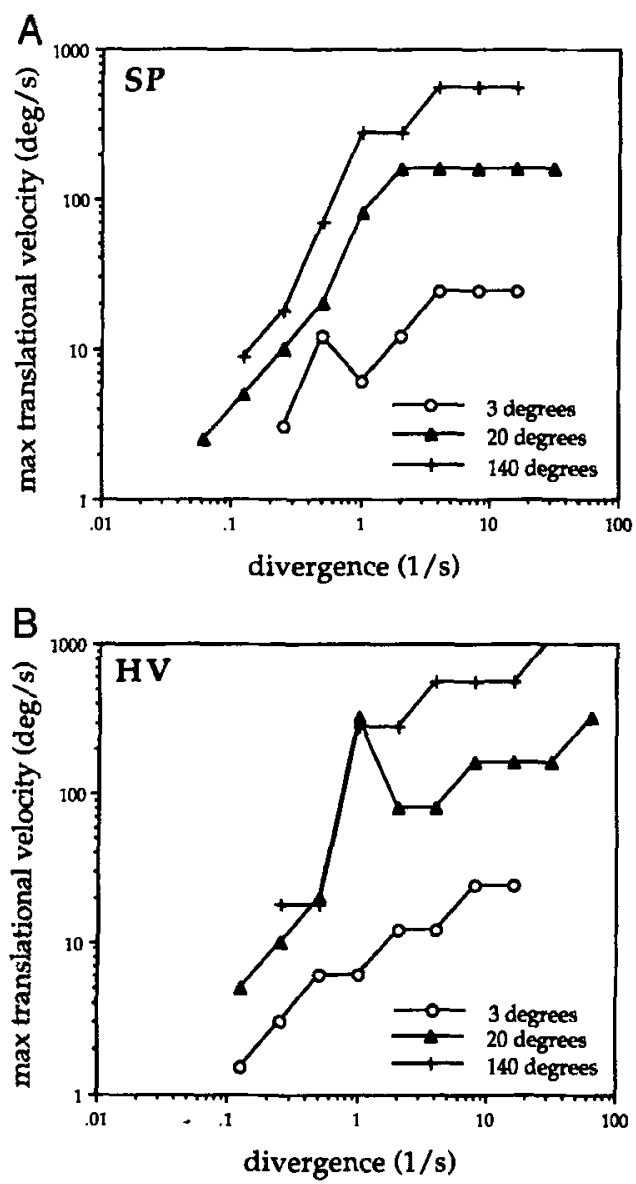

Figure 6. The maximum translational velocity as a function of divergence for three different field sizes. (A) Results for Subject S.P. (B) Results for Subject H.V.

size on the height of the noise levels. Apparently subjects perform just as well with only peripheral information.

In Figures 8A-8B we can observe the same phenomenon. It shows the maximum translational velocity as a function of divergence for Subjects S.P. and H.V., respectively. The relative area of the gap had no influence whatsoever on the maximum translational velocity that could be used.

\section{DISCUSSION}

We addressed the question of whether the extraction of information from first-order flow fields depends on the field size. Such a dependency would not be surprising a priori, because of the entirely different information needed in performing tasks such as, for instance, navigation through space and object recognition. We suggested in our introduction that the recognition of objects and shapes involves small optical flow fields and requires high resolution of the elements at low speeds. On the other hand, orientation and navigation involve large fields and require the flowfield elements to move at high speed. Psychophysical experiments with different field sizes (with a diameter of $3^{\circ}$, $20^{\circ}$, or $140^{\circ}$ of visual angle) were performed to investigate whether parameter ranges vary with stimulus size. Subjects had to indicate the polarity of a diverging flow field in the presence of a translational velocity, a task not directly associated with either navigation or object recognition. We found qualitatively similar noise thresholds for all field sizes.

The difference in thresholds that we did find is a scaling, meaning that we get the same results for all stimulus sizes if we express the thresholds in units of stimulus diameter. Our findings support the viewing-distance invariance found by van de Grind, Koenderink, and van Doorn (1992) for zeroth-order flow fields (moving Julesz patterns). These authors found that performance is constant irrespective of viewing distance for a relatively wide range of field sizes. Werkhoven and Koenderink (1993), however, concluded that visual size invariance does not hold for speed of rotation. Therefore, we did not expect to find viewing-distance invariance for expansion. We feel that the difference between their result and ours is due to a different set-up and task (discrimination of the speed of rotation, in their case), and not due to a difference between expansion and rotation. In previous experiments with stimuli that were $20^{\circ}$ of visual angle in diameter (te Pas et al.,
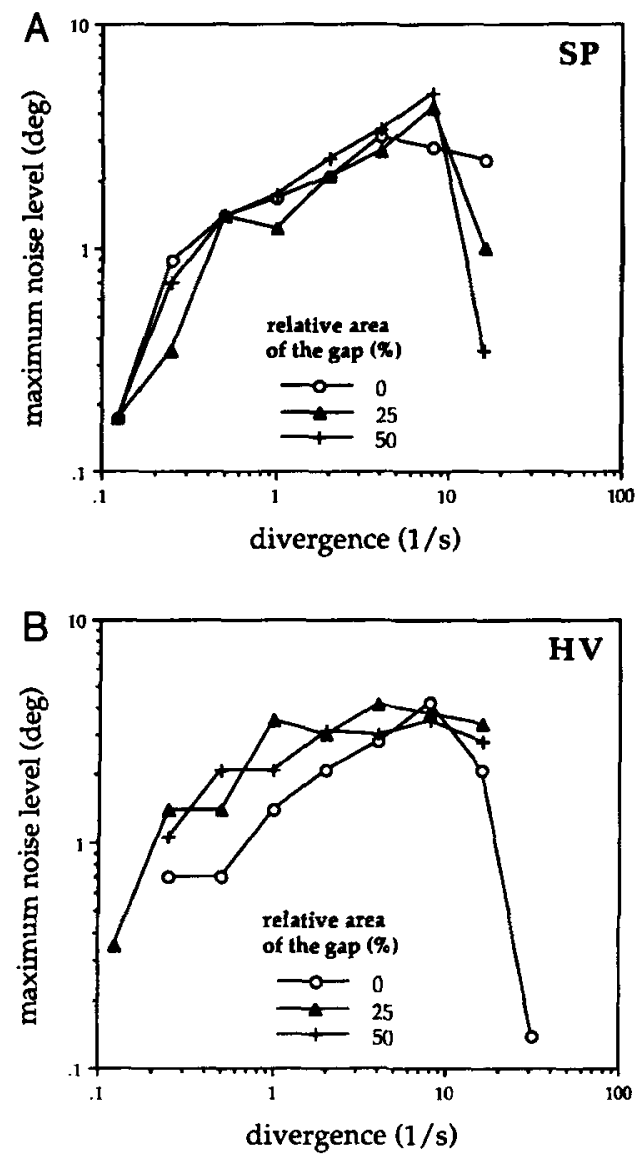

Figure 7. The maximum noise level (SD of the Gaussian perturbation vector) as a function of divergence for three different gap sizes. (A) Results for Subject S.P. (B) Results for Subject H.V. 

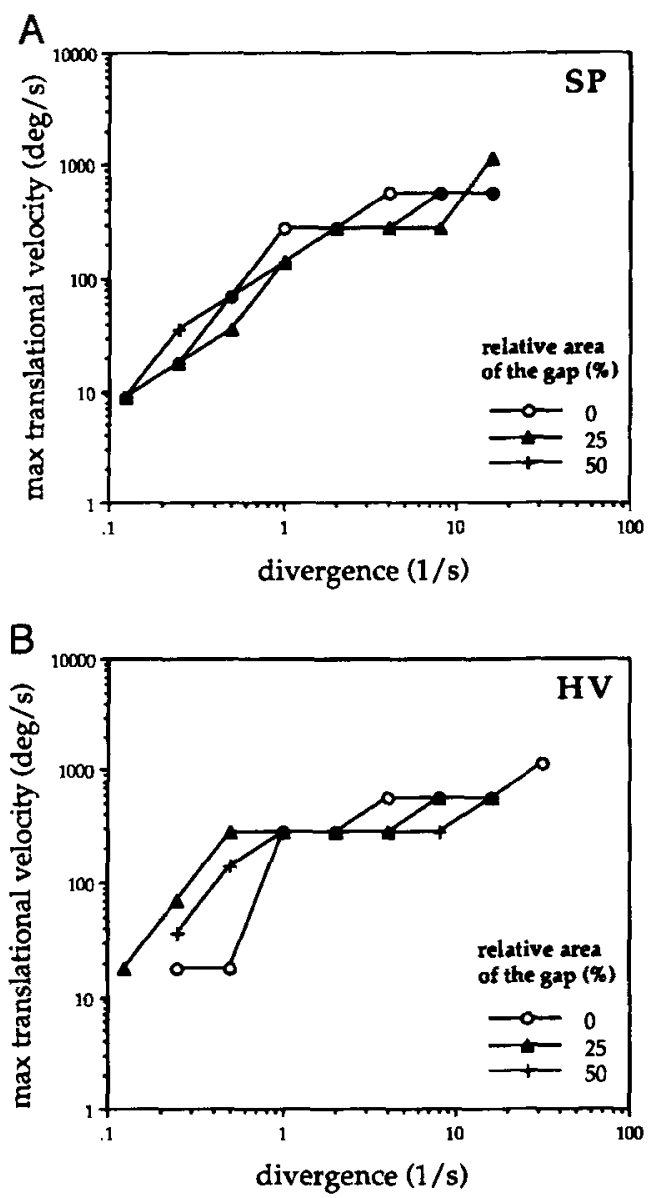

Figure 8: The maximum translational velocity as a function of divergence for three different gap sizes. (A) Results for Subject S.P. (B) Results for Subject H.V.

1996), and in a control experiment with stimuli of $3^{\circ}$ of visual angle in diameter, we found no differences between rotation and expansion.

We also ran psychophysical experiments to test whether subjects could indicate the polarity of divergence when only peripheral information is available. In this experiment we used a stimulus with a diameter of $140^{\circ}$ of visual angle. We introduced a variable central gap of up to $125^{\circ}$ in diameter to eliminate foveal information. Thresholds for detecting the polarity of divergence in the presence of translational velocity remained the same for all gap sizes (ranging from $0^{\circ}$ to $125^{\circ}$ of visual angle). Thus, for this task, subjects appeared not to be bothered by the lack of foveal information. This finding supports the results found by Crowell and Banks (1993). They concluded, using a radial pattern at different eccentricities, that foveal information is not more important than peripheral information. Increasing the field size (as we did in the first experiment) effectively increases the amount of peripheral information relative to the amount of foveal information. This helps the performance of our subjects. Eliminating foveal information (as we did in the second experiment) did not in- fluence the performance of our subjects at all. Combining both experiments, we can conclude that subjects use largely peripheral information for this task. This finding is consistent with results of Lappin, Norman, and Mowafy (1991). They showed that the detection of coherent expansion of dots that were located at $5^{\circ}$ is easy, even when embedded in a background of translating dots.

Other experiments (Freeman \& Harris, 1992; Kappers, te Pas, \& Koenderink, 1993; Tanaka, Fukada, \& Saito, 1989) show that the directional relationship of the velocity vectors contains the most important information, rather than the velocity gradient or the exact value of the first order component. In our experiments, the importance of the peripheral information suggests that subjects mainly use velocity vectors on the left and on the right side of the stimulus, where the difference between them is largest. Subjects could, for instance, subtract two velocity vectors on the extreme left and extreme right side of the stimulus. However, this strategy predicts a linear relation between the divergence and the maximum noise level, and we did not find that. If subjects use the maximum angle between two velocity vectors in the stimulus, peripheral information would also be most important. However, when we scaled the stimulus, this angle did not change, but the performance of our subjects improved. Moreover, we know from previous experiments (te Pas et al., 1996) that although this strategy can explain some results, it does not tell the whole story. Thus, the strategy that subjects use must be more complicated than that, and is as yet not known.

We did not find qualitatively different behavior in different parameter ranges in our experiments. Thus, on the question of whether the visual system has specialized parameter ranges for different tasks, our results are not conclusive. We feel that the question of whether there are different processes for shape from motion and for orientation and navigation is an interesting one, and we hope that future research will shed more light on this problem. However, the results strongly support the viewing-distance invariance hypothesis (van de Grind et al., 1992). Subjects show the same behavior over a surprisingly large range of field sizes. Moreover, results show that foveal information is not essential for this task.

\section{REFERENCES}

Crowell, J. A., \& Banks, M. S. (1993). Perceiving heading with different retinal regions and types of optic flow. Perception \& Psychophysics, 53, 325-337.

Dijkstra, T. M. H., Cornilleau-Pérès, V., Gielen, C. C. A. M., \& Droulez, J. (1995). Perception of three-dimensional shape from egoand object-motion: Comparison between small and large field stimuli. Vision Research, 35, 453-462.

Freeman, T. C. A., \& Harris, M. G. (1992). Human sensitivity to expanding and rotating motion: Effects of complementary masking and directional structure. Vision Research, 32, 81-87.

GiBSON, J. J. (1950). The perception of the visual world. Boston: Houghton Mifflin.

Gibson, J. J. (1966). The senses considered as perceptual systems. Boston: Houghton Mifflin.

Hubel, D. H., \& Wiesel, T. N. (1974). Uniformity of monkey striate cortex: A parallel relationship between field size, scatter and magnification factor. Journal of Comparative Neurology, 158, 295-306. 
Kappers, A. M. L., te Pas, S. F., \& Koenderink, J. J. (1993). Detection of divergence in optic-flow fields. Perception, 22(Suppl. 83a).

Kappers, A. M. L., van Doorn, A. J., \& Koenderink, J. J. (1994). Detection of vorticity in optical flow fields. Journal of the Optical Society of America $A, 11,48-54$.

KoENDERINK, J. J. (1986). Optic flow. Vision Research, 26, 161-180.

KOENDERINK, J. J., \& VAN DOORN, A. J. (1975). Invariant properties of the motion parallax field due to the movement of rigid bodies relative to an observer. Optica Acta, 22, 773-791.

KoENDERINK, J. J., \& VAN DOORN, A. J. (1976). Local structure of movement parallax of the plane. Journal of the Optical Society of America, 66, 717-723.

Koenderink, J. J., van Doorn, A. J., \& VAN DE Grind, W. A. (1985). Spatial and temporal parameters of motion detection in the peripheral visual field. Journal of the Optical Society of America A, 2, 252-259.

LapPin, J. S., Norman, J. F., \& Mowafy, L. (1991). The detectability of geometric structure in rapidly changing optical patterns. Perception, 20, 513-528.

MCKEE, S. P., \& NaKayama, K. (1984). The detection of motion in the peripheral visual field. Vision Research, 24, 25-32.

Orban, G. A., Van Calenbergh, F., De Bruyn, B., \& Maes, $H$. (1985). Velocity discrimination in central and peripheral visual field. Journal of the Optical Society of America A, 2, 1836-1847.

TANAKa, K., Fukada, Y., \& Saito, H.-A. (1989). Underlying mechanisms of the response specificity of expansion/contraction, and rota- tion cells in the dorsal part of the medial superior temporal area of the macaque monkey. Journal of Neurophysiology, 62, 642-656.

te Pas, S. F., Kappers, A. M. L., \& Koenderink, J. J. (1996). Detection of first-order structure in optic flow fields. Vision Research, 36, 259-270.

VAN DE Grind, W. A., Koenderink, J. J., \& VAN DOORN, A. J. (1992).

Viewing-distance invariance of movement detection. Experimental Brain Research, 91, 135-150.

van DE Grind, W. A., van DoOrn, A. J., \& Koenderink, J. J. (1983). Detection of coherent movement in peripherally viewed random-dot patterns. Journal of the Optical Society of America, 73, 1674-1683.

van DoORn, A. J., \& KoEnderinK, J. J. (1982a). Spatial properties of the visual detectability of moving spatial white noise. Experimental Brain Research, 45, 189-195.

VAN Doorn, A. J., \& Koenderink, J. J. (1982b). Temporal properties of the visual detectability of moving spatial white noise. Experimental Brain Research, 45, 179-188.

WARREN, W. H., \& KURTZ, K. J. (1992). The role of central and peripheral vision in perceiving the direction of self-motion. Perception \& Psychophysics, 51, 443-454.

Werkhoven, P., \& Koenderink, J. J. (1993). Visual size invariance does not apply to geometric angle and speed of rotation. Perception, 22, 177-184.

(Manuscript received May 9, 1995; revision accepted for publication August 10, 1995.) 